\title{
Gegen das Zittern: Essenziellen Tremor und Parkinson-Tremor mit Ultraschall behandeln
}

Berlin - Einen Tee einschenken oder Haare kämmen - wenn die Hände unkontrollierbar zittern, werden einfachste Aufgaben zur Herausforderung. Patienten mit solchen Symptomen leiden oft an einem essenziellen oder an einem Parkinson-Tremor, chronisch fortschreitenden Bewegungsstörungen mit unwillkürlichem Zittern. Die Standardtherapie mit Medikamenten ist nur selten ausreichend und mit erheblichen Nebenwirkungen behaftet. Bei der operativen Therapie, der sogenannten „tiefen Hirnstimulation“ (THS), werden Elektroden in der Tiefe des Gehirns platziert, was mit einer Eröffnung des Schädels einhergeht. Eine neue Therapie, bei der die tief im Gehirn liegenden Störherde mittels Magnetresonanztomografie-gesteuertem fokussiertem Ultraschall (MRgFUS) ausgeschaltet werden, kann die Beschwerden auch ohne offene Chirurgie deutlich lindern. Eine aktuelle Studie - in der Zeitschrift „Neurology“ erschienen - belegt, dass das Zittern bei den Teilnehmern auch 3 Jahre nach dem Eingriff noch deutlich verbessert war. Die Deutsche Gesellschaft für Ultraschall in der Medizin (DEGUM) empfiehlt den Einsatz der neuen Methode, da sie schonend und präzise angewandt werden kann.

Ein Tremor tritt am häufigsten an Händen oder Armen auf, aber auch der Rumpf oder der Kopf können betroffen sein. „Die Bewegungsstörung entsteht im Groß- und Zwischenhirn in Bereichen, die an der Bewegungssteuerung beteiligt sind“, sagt DEGUM-Experte Professor Dr. Ullrich Wüllner, Leiter der Sektion Bewegungsstörungen an der Klinik für Neurologie des Universitätsklinikums Bonn. Die Aktivität dieser Hirnareale ist zwar prinzipiell über Medikamente beeinflussbar, die Mittel wirken jedoch längst nicht bei jedem Patienten.
Die operative Therapie war bislang ein stereotaktischer Eingriff, bei dem die betreffenden Hirnareale mithilfe fest implantierter Elektroden und elektrischen Impulsen aus einem Batterie-Aggregat - ähnlich einem Herzschrittmacher - moduliert werden. Bei diesem als tiefe Hirnstimulation bezeichneten Verfahren muss jedoch der Schädel des Patienten geöffnet werden, dadurch entsteht die Gefahr von Hirnblutungen oder Infektionen.

Eine schonendere Alternative bietet der Magnetresonanztomografie-gesteuerte fokussierte Ultraschall (MRgFUS): „Das neuartige Verfahren funktioniert ohne operativen Eingriff. Es legt von außen gezielt nur diejenigen Hirnareale lahm, die für das Zittern verantwortlich sind“, erläutert Wüllner. „Die Ultraschallwellen werden genau auf den Zielpunkt gesendet und dort wie bei einem Brennglas gebündelt.“ Eine MRT-gesteuerte Neuronavigation stellt dabei sicher, dass nur der gewünschte Bereich im Gehirn inaktiviert wird. Zudem erfassen die behandelnden Ärzte bereits während der Behandlung die optimale Tremor-Kontrolle.

Die aktuelle Studie aus der angesehenen Zeitschrift „Neurology“ bestätigt die Langzeitwirkung des Verfahrens. Forscher um Dr. Casey H. Halpern von der Stanford-Universität haben das moderne Ultraschallverfahren bei 76 Patienten mit therapieresistentem essenziellem Tremor angewendet. Selbst 3 Jahre nach der Behandlung war das Zittern bei der Hälfte der Studienteilnehmer noch deutlich verbessert. Auch Behinderungen und die Lebensqualität, die durch die chronische Bewegungsstörung vor dem Eingriff stark eingeschränkt war, hatten sich bei vielen Patienten noch gebessert (56 und 42\%). Da der Eingriff trotz des schonenden Verfahrens - eine umschriebene, dauerhafte Schädigung im Gehirngewebe hinterlässt, können als $\mathrm{Ne}$ benwirkungen Gefühls-, Gang- und Gleichgewichtsstörungen auftreten. Im Vergleich zu den Nebenwirkungen eines operativen Eingriffs sind diese jedoch eher gering.

DEGUM-Experte Wüllner macht selbst positive Erfahrungen mit dem fokussierten Ultraschallverfahren: Seit 1,5 Jahren führt er dieses in seiner Bonner NeurologieAbteilung durch - und konnte damit bereits 35 Patienten erfolgreich behandeln. „Bei Parkinson-Patienten, deren Tremor medikamentös nicht in den Griff zu bekommen war, zeigt die Therapie sehr gute, anhaltende Erfolge“, sagt der Neurologe. Bei Patienten mit essenziellem Tremor tritt der Tremor bei etwa $30 \%$ der Patienten im Verlauf abgeschwächt wieder auf. Dieser Langzeitverlauf wird auch in Bonn im Rahmen einer Beobachtungsstudie am Deutschen Zentrum für neurodegenerative Erkrankungen (DZNE) genau studiert. Aufgrund der guten Erfahrungswerte, die nun erstmals auch langfristig nachgewiesen werden konnten, fordert die DEGUM eine Aufnahme der modernen Ultraschalltherapie in den Katalog der gesetzlichen Krankenkassen. Die Kosten dafür werden von diesen derzeit noch nicht regelhaft übernommen.

\section{HINTERGRUND}

Link zum Abstract der Studie: https:// n.neurology.org/content/93/24/e2284 